\title{
All for One: Feature Combination for Highly Channel-Degraded Speech Activity Detection
}

\author{
Martin Graciarena ${ }^{1}$, Abeer Alwan ${ }^{4}$, Dan Ellis ${ }^{5,2}$, Horacio Franco ${ }^{1}$, Luciana Ferrer ${ }^{1}$, John H.L. \\ Hansen $^{3}$, Adam Janin ${ }^{2}$, Byung-Suk Lee ${ }^{5}$, Yun Lei ${ }^{1}$, Vikramjit Mitra ${ }^{1}$, Nelson Morgan ${ }^{2}$, Seyed Omid \\ Sadjadi $^{3}$, TJ Tsai ${ }^{2}$, Nicolas Scheffer ${ }^{1}$, Lee Ngee Tan ${ }^{4}$, Benjamin Williams ${ }^{1}$ \\ ${ }^{1}$ Speech Technology and Research Laboratory, SRI International, Menlo Park, CA, USA \\ ${ }^{2}$ International Computer Science Institute (ICSI), Berkeley, CA, USA \\ ${ }^{3}$ Center for Robust Speech Systems (CRSS), U.T. Dallas, Richardson, TX, USA \\ ${ }^{4}$ Speech Processing and Auditory Perception Lab., Univ. of California, Los Angeles, CA, USA \\ ${ }^{5}$ LabROSA, Columbia University, NY, USA \\ martin@speech.sri.com
}

\begin{abstract}
Speech activity detection (SAD) on channel transmissions is a critical preprocessing task for speech, speaker and language recognition or for further human analysis. This paper presents a feature combination approach to improve SAD on highly channel degraded speech as part of the Defense Advanced Research Projects Agency's (DARPA) Robust Automatic Transcription of Speech (RATS) program. The key contribution is the feature combination exploration of different novel SAD features based on pitch and spectro-temporal processing and the standard Mel Frequency Cepstral Coefficients (MFCC) acoustic feature. The SAD features are: (1) a GABOR feature representation, followed by a multilayer perceptron (MLP); (2) a feature that combines multiple voicing features and spectral flux measures (Combo); (3) a feature based on subband autocorrelation (SAcC) and MLP postprocessing and (4) a multiband comb-filter F0 (MBCombF0) voicing measure. We present single, pairwise and all feature combinations, show high error reductions from pairwise feature level combination over the MFCC baseline and show that the best performance is achieved by the combination of all features.
\end{abstract}

Index Terms: speech detection, channel-degraded speech, robust voicing features

\section{Introduction}

Speech activity detection (SAD) on noisy channel transmissions is a critical preprocessing task for speech, speaker and language recognition or for further human analysis. SAD tackles the problem of separation between speech and background noises and channel distortions such as spurious tones, etc.

Numerous methods have been proposed for speech detection. Some simple methods are based on comparing the frame energy, zero crossing rate, periodicity measure, or spectral entropy with a detection threshold to make the speech/nonspeech decision. More advanced methods include long-term spectral divergence measure [1, 2], amplitude probability distribution [3], and lowvariance spectrum estimation [4].

This paper presents a feature combination approach to improve speech detection performance. The main motivation is to improve the baseline acoustic feature performance with different novel pitch and spectro-temporal processing features by exploring the complementary information from the presence of a pitch structure or from a different spectro-temporal representation. We combine an MFCC acoustic feature with four speech activity detection features: (1) a GABOR feature representation followed by a multilayer perceptron that produces a speech confidence measure; (2) a Combo feature that combines multiple voicing features and a spectral flow measure; (3) a feature based on subband autocorrelation (SAcC) and MLP postprocessing and (4) a multiband comb-filter F0 (MBCombF0) voicing measure estimated from a multiple filterbank representation. We present speech detection results for highly channel-degraded speech data collected as part of the DARPA RATS program. We show gains from feature level combination, resulting in significant error reductions over the MFCC baseline.

The RATS program aims at the development of robust speech processing techniques for highly degraded transmission channel data, specifically for $\mathrm{SAD}$, for speaker and laguage identification and keyword spotting. The data was collected by the Linguistic Data Consortium (LDC) by retransmitting conversational telephone speech through eight different communication channels [12] using multiple signal transmitters/transceivers, listening station receivers and signal collection and digitization apparatus. The RATS rebroadcasted data is unique in that it contains a wide array of real transmission distortions such as: band limitation, strong channel noises, nonlinear speech distortions, frequency shifts, high energy non transmission bursts, etc.

The SAD system is based on a smoothed log likelihood ratio between a speech Gaussian mixture model (GMM) and a background GMM. The SAD model is similar to the one presented by $\mathrm{Ng}$ et. al. [11], however we used different model and likelihood smoothing parameters. The long span feature and dimensionality reduction technique differ from the one from $\mathrm{Ng}$, instead of Heterosedastic linear discrimination (HLDA) we used a Discrete cosine transform (DCT) technique. In Ng's paper the DCT component is used but on the MLP SAD subcomponent. Finally the types of features differ as well. In our work we present the standard acoustic features as well as four different types of features ranging from spectro-temporal to voicing derived features, whereas $\mathrm{Ng}$ 's paper a combination of standard acoustic feature and cortical based features. 


\section{Features Description}

This section describes specific aspects of each of the four SADspecific features: GABOR, Combo, SAcC and MBCombF0.

\subsection{GABOR Feature}

The GABOR with MLP feature is computed by processing a Mel spectrogram by 59 real-valued spectro-temporal filters covering a range of temporal and spectral frequencies. Each of these filters can be viewed as correlating the time-frequency plane with a particular ripple in time and frequency. Because some of these filters yield very similar outputs for neighboring spectral channels, only a subset of 449 GABOR features is used for each time frame. As the final preprocessing step, mean and variance normalization of the features over the training set is performed. GABOR features are described in [5].

Next, a MLP is trained to predict speech/nonspeech labels given 9 frames of the 449 GABOR features, or 4,041 inputs. The MLP uses 300 hidden units and 2 output units. The size of the hidden layer is chosen so that each MLP parameter has approximately 20 training data points. Although the MLP is trained with a softmax nonlinearity at the output, during feature generation the values used are the linear outputs before the nonlinearity. The resulting 2 outputs are then mean and variance normalized per file, and used as the input features to the classification backend.

\subsection{Combo Feature}

This section describes the procedure for extracting a 1dimensional feature vector that has been shown to possess great potential for speech/non-speech discrimination in harsh acoustic noise environments [6]. This "combo" feature is efficiently obtained from a linear combination of four voicing measures as well as the perceptual spectral flux (SF). The perceptual SF and periodicity are extracted in the frequency domain, whereas the harmonicity, clarity, and prediction gain are time domain features.

The combo feature includes the following: (1) Harmonicity (also known as harmonics-to-noise ratio) is defined as the relative height of the maximum autocorrelation peak in the plausible pitch range. (2) Clarity is the relative depth of the minimum average magnitude difference function (AMDF) valley in the plausible pitch range. Computing the AMDF from its exact definition is costly; however, it has been shown [7] that the AMDF can be derived (analytically) from the autocorrelation. (3) Prediction gain is defined as the ratio of the signal energy to the linear prediction (LP) residual signal energy. (4) Periodicity, in the short-time Fourier transform domain, is the maximum peak of the harmonic product spectrum (HPS) [8] in the plausible pitch range. (5) Perceptual SF measures the degree of variation in the subjective spectrum across time. In short-time frames, speech is a quasistationary and slowly varying signal, that is, its spectrum does not change rapidly from one frame to another.

After extracting the features, a 5-dimensional vector is formed by concatenating the voicing measures along with the perceptual SF. Each feature dimension $f_{i}$ is normalized by its mean and variance over the entire waveform. The normalized 5dimensional feature vectors are linearly mapped into a 1dimensional feature space represented by the most significant eigenvector of the feature covariance matrix. This is realized through principal component analysis (PCA), and by retaining the dimension that corresponds to the largest eigenvalue. The 1dimensional feature vector is further smoothed via a 3-point median filter and passed to the next stage for speech activity detector.

\subsection{MBCombF0 Feature}

The voicing feature is the estimated degree of voicing of each frame computed by the MBCombF0 algorithm, which is a modification of the correlogram-based F0 estimation algorithm described in [9]. The processing sequence of the MBCombF0 is the following. A frame length of $100 \mathrm{~ms}$ is used. First, the input signal is downsampled to $8 \mathrm{kHz}$ and split into four subbands that cover 0 to $3.4 \mathrm{kHz}$. Each subband has a $1-\mathrm{kHz}$ bandwidth and overlaps the adjacent filter by $0.2 \mathrm{kHz}$. Envelope extraction is then performed on each subband stream, followed by multichannel comb-filtering with comb filters of different interpeak frequencies.

Next, reliable comb-channels are selected individually for each subband using a 3 -stage selection process. The first selection stage is based on the comb-channel's harmonic-tosubharmonic-energy ratio in the respective subband, those with a peak magnitude greater than one. In the second stage, combchannels and their corresponding subharmonic channels (with an interpeak frequency that is half of the former) are retained if both are present in this initial selected set. In the final selection stage, channels whose maximum autocorrelation peak location (computed from their comb-filtered outputs) is close to their corresponding comb-filters' fundamental period are selected. A subband summary correlogram is then derived from the weighted average of selected energy-normalized autocorrelation functions. Finally, the four subband summary correlograms are combined using a subband reliability weighting scheme to form the multiband summary correlogram. The weighting of each subband depends on its maximum harmonic-to-subharmonicenergy ratio and the number of the subband summary correlogram whose maximum peak location is similar to its own. Time-smoothing is then applied to the multiband summary correlogram as described in [9], and the maximum peak magnitude of the resulting summary correlogram is the $\mathrm{MBC}$ ombF0 voicing feature extracted.

\subsection{SAcC Feature}

The SAcC feature (for Subband Autocorrelation Classification) [10] is derived from our noise-robust pitch tracker. SAcC involves an MLP classifier trained on subband autocorrelation features to estimate, for each time frame, the posterior probability over a range of quantized pitch values, and one "nopitch" output. We trained a RATS-specific MLP by using the consensus of conventional pitch trackers applied to the clean (source) signal to create a ground truth for each of the noisy (received) channels; we trained a single MLP for all channels. For this system, we used only the "no-pitch" posterior as a feature to indicate the absence of voiced speech in the signal frame.

\subsection{Feature Figures}

Figure 1 shows a plot of the channel-degraded waveform for channel A, spectrogram, labels, and the GABOR, Combo, SAcC and $\mathrm{MBCombF0}$ feature outputs per frame. Rectangles superimposed on the waveform highlight the speech regions. 
Notice the highly channel-degraded waveform and low signal to noise ratio (SNR). The labels are 1 for speech, 0 for non-speech and -1 for no transmission regions. The no transmission regions are high energy white noise type of sounds interleaved between valid signal transmissions. It is clear the GABOR features are much smoother with a long time span. Other SAD features are frame-based so they have a more dynamic behavior. However they all achieve good detection of the speech regions. Interestingly the three voicing based features provide somewhat different outputs.
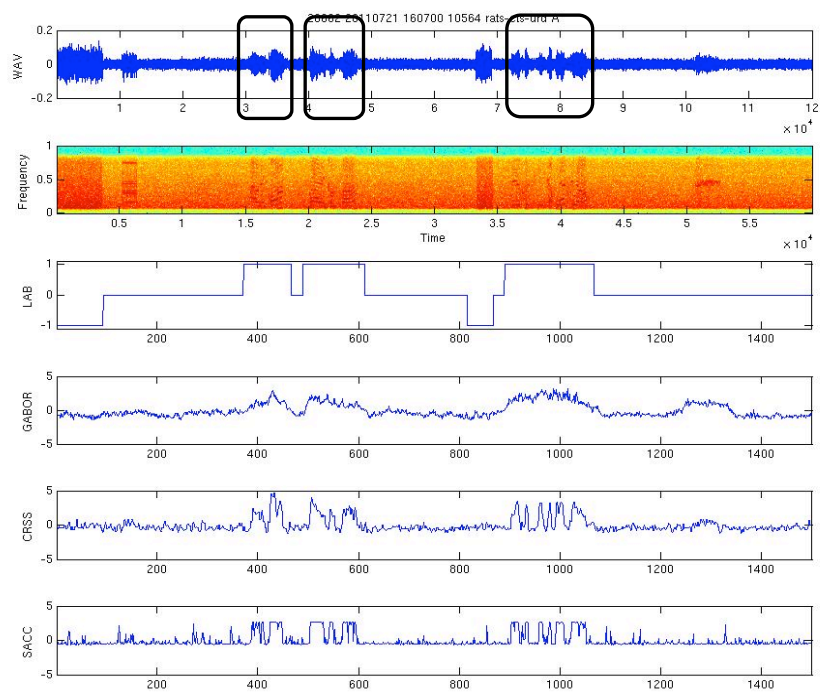

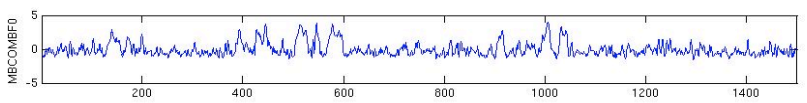

Figure 1: Waveform, spectrogram, ground truth speech and nonspeech labels, GABOR, Combo, SAcC and MBCombF0

features. Speech regions marked in black rectangles.

\section{SAD Description}

The SAD system is based on a frame-based smoothed likelihood ratio (LLR) setup. The LLR is computed between speech and nonspeech Gaussian mixture models (GMM). Then the LLR is smoothed with a multiple window median filter of length 51 frames. Finally the speech regions are obtained from the smoothed LLR frames which are higher than a given threshold. No padding was used to artificially extend the speech regions.

Additionally we used long range modeling using a 1dimensional Discrete Cosine Transform (DCT). For each feature dimension we first created a window of multiple frames. Next we computed the DCT transform and only preserved a subset of the initial DCT coefficients to obtain the desired number of features. This results in a low-dimensional representation of the feature modulation within the multi-frame window. We found that a 30 frame window was optimal for most features. We then concatenated the DCT dimensionality-reduced features for all dimensions and applied waveform level mean and variance normalization.

For most of the experiments we used 256 Gaussian full covariance models for speech and nonspeech classes. We trained channel dependent models, therefore at test time we ended up with 16 models, 8 for speech and 8 for nonspeech. When testing SAD features we used 32 Gaussian full covariance models due to their reduced feature dimension. During testing we obtained the LLR from the numerator obtained as the sum of the log probability of the speech models given the current feature and the denominator obtained from the sum of the log probability of the nonspeech models given the current feature.

\section{Experiments}

\subsection{Data Description}

This section discusses speech detection in RATS data. We present the results of each feature in isolation and then the feature level combination results.

The data used belongs to the LDC collections for the DARPA RATS program LDC2011E86, LDC2011E99 and LDC2011E111. We tested on the Dev-1 and Dev-2 sets. These two devsets contain similar data but we found Dev-2 to contain speech at lower SNR. The data was annotated with speech and background labels. More details are presented in Walker and Strassel [12].

The audio data was retransmitted using a multilink transmission system designed hosted at LDC. Eight combinations of analog transmitters and receivers were used covering a range of carrier frequencies, modes and bandwidths, from $1 \mathrm{MHz}$ amplitude modulation to $2.4 \mathrm{GHz}$ frequency modulation.

The audio material for retransmission was obtained from existing speech corpora such as Fisher English data, Levantine Arabic telephone data and RATS program specific collections, which included speech in several languages such as English, Pashto, Urdu, Levantine Arabic, etc.

\subsection{Error Computation}

The equal error rate (EER) was computed from two error measures using SAIC's RES engine which is the official SAD scoring for the RATS program. One error measure is the probability of missing speech (Pmiss), and the second is the likelihood of wrongly hypothesizing speech (Pfa). These are computed as follows:

Pmiss $=$ total_missed_speech / total_scored_speech $\mathrm{Pfa}=$ total_false_accept_speech / total_scored_nonspeech

where total_missed_speech is the duration of the undetected speech regions, and total_scored_speech is the duration of all the speech regions. Total_false_accept_speech is the duration of the false hypothesized speech segments, and total_scored nonspeech is the total duration of the nonspeech regions.

\subsection{Speech Detection Results}

Table 1 shows the \% EER for different input features on Dev-1 and Dev-2 sets. We first tested all the features in isolation. Next in Table 2 we performed a two way combination between MFCC and each of the other SAD features. For example in the first case we appended the $40 \mathrm{dim}$ MFCC to a 4-dimensional GABOR feature, resulting in a 44 dimensional feature vector. Finally on Table 2 we performed full feature combination between MFCC and the four SAD features resulting in a 56 dimensional feature vector. On Table 3 we present the channel specific results from the all feature combination system. Notice that channel D is missing, as it was officially excluded from scoring. 
Table 1: Single Feature Speech Detection \% EER Results.

\begin{tabular}{|l|c|c|c|c|}
\hline $\begin{array}{l}\text { Input } \\
\text { Features }\end{array}$ & $\begin{array}{c}\text { Feat } \\
\text { Dim }\end{array}$ & $\begin{array}{c}\text { Model } \\
\text { Gauss }\end{array}$ & $\begin{array}{c}\text { \% EER } \\
\text { Dev-1 }\end{array}$ & $\begin{array}{c}\text { \% EER } \\
\text { Dev-2 }\end{array}$ \\
\hline MFCC (baseline) & 40 & 256 & 2.05 & 2.70 \\
\hline GABOR & 4 & 32 & 4.00 & 5.45 \\
\hline Combo & 4 & 32 & 4.20 & 4.75 \\
\hline SAcC & 4 & 32 & 4.75 & 4.90 \\
\hline MBCombF0 & 4 & 32 & 4.10 & 6.15 \\
\hline
\end{tabular}

Analyzing the results in Table 1 where we first compared the performance of the five isolated features. We used the performance of the MFCC feature with DCT processing as the baseline. It obtained a very low EER on both Dev-1 and Dev-2 sets. Next we compared the other four SAD features in isolation. On Dev-1 GABOR achieves the lowest EER, followed by MBCombF0 and finally Combo and SAcC. However on Dev-2 the best feature is Combo, followed by SAcC, GABOR and MBCombF0. This reveals that some features might be able to capture better the specific types of distortions in one set but fail to generalize to the other set. The increased errors on Dev-2 might be due to the fact that SNRs are lower than on Dev-1.

Table 2: Feature Combination Speech Detection \% EER Results.

\begin{tabular}{|l|c|c|c|c|}
\hline $\begin{array}{c}\text { Input } \\
\text { Features }\end{array}$ & $\begin{array}{c}\text { Feat } \\
\text { Dim }\end{array}$ & $\begin{array}{c}\text { Model } \\
\text { Gauss }\end{array}$ & $\begin{array}{c}\text { \% EER } \\
\text { Dev-1 }\end{array}$ & $\begin{array}{c}\text { \% EER } \\
\text { Dev-2 }\end{array}$ \\
\hline MFCC + GABOR & 44 & 256 & 1.70 & 2.50 \\
\hline MFCC + Combo & 44 & 256 & 1.85 & 2.40 \\
\hline MFCC + SACC & 44 & 256 & 1.90 & 2.45 \\
\hline MFCC + MBCombF0 & 44 & 256 & 1.65 & 2.45 \\
\hline MFCC + All SAD & 56 & 256 & 1.55 & 2.10 \\
\hline
\end{tabular}

Analyzing the results on Table 2 we found important error reductions on both sets when combining one SAD feature with the MFCC feature compared to the baseline MFCC performance. On Dev-1 the best pairwise combination is with MBCombF0 followed by the combination with GABOR. Interestingly this reverses the order of performance from Table 1 of each of these features in isolation. The combination with Combo and SAcC also produces error reductions compared to the baseline. On Dev-2 the best pairwise combination with Combo feature, followed closely by the combination with SAcC, MBCombF0 and finally GABOR. This trend in Dev-2 additionally shows that these different features in combination and over different testsets produce different gains, therefore there is hope that the combination of them will result in improved performance overall.

Finally the best performance is found from the all feature combination on both devsets. On Dev-1 the relative gain from the all feature combination system over the MFCC baseline is $24.3 \%$ and over the best pairwise combination is $6.0 \%$. On Dev2 the relative gain from the all feature combination system over the MFCC baseline is $22.2 \%$ (about the same as on Dev-1) and over the best pairwise combination is $12.5 \%$. This means that each SAD feature provides different complementary information to the baseline MFCC feature. This is a very relevant result as three out of the four SAD features (Combo, SAcC and MBCombF0) aim at capturing voicing information. Since each of these features approach the problem from a different perspective and use different processing techniques, which in complementary information.
Table 3: \% EER Results by Channel on Dev-1 from MFCC+All SAD Feature System.

\begin{tabular}{|c|c|c|c|c|c|c|c|}
\hline $\begin{array}{c}\text { Input } \\
\text { Feature }\end{array}$ & A & B & C & E & F & G & H \\
\hline $\begin{array}{c}\text { MFCC + } \\
\text { All SAD }\end{array}$ & 2.25 & 2.40 & 2.35 & 2.00 & 2.45 & 0.70 & 1.20 \\
\hline
\end{tabular}

Finally in Table 3 we present the channel specific results on Dev-1 from the all feature combination system which performed best on Table 2. The best performance is achieved on channel G, followed by channel $\mathrm{H}$ and the rest of the channels with similar performance overall. On channel $\mathrm{G}$ the signal is very clear and $\mathrm{SNR}$ is higher compared to other channels. Channel $\mathrm{H}$ also contains come high SNR recordings. The other channels contain different types of distortions and vary in SNR and speech degradation types. Overall the performance is similar in those highly degraded channels which reveal a consistent behavior of the developed SAD. However performance on those degraded channels lag behind channels $\mathrm{G}$ and $\mathrm{H}$, which reveal that there is still work to do to minimize that difference.

\section{Conclusions}

Our feature combination approach results in a highly accurate speech detector despite high degradation by channel noise and transmission distortions. We found significant gains from combining a MFCC acoustic feature with four speech activity detection features: GABOR, Combo, SAcC and MBCombF01. These SAD features differ in their processing techniques, one is based on spectro-temporal processing and the other three are based on voicing measure estimation. Their different processing techniques and approaches result in different performance over two different test sets. These results result in important gains when combining with the baseline feature. Finally we found important gains in performance when combining all the features, which is the major benefit from the feature combination exploration explored in this paper.

\section{Acknowledgements}

This material is based on work supported by the Defense Advanced Research Projects Agency (DARPA) under Contract No. D10PC20024. Any opinions, findings, and conclusions or recommendations expressed in this material are those of the author(s) and do not necessarily reflect the view of the DARPA or its Contracting Agent, the U.S. Department of the Interior, National Business Center, Acquisition \& Property Management Division, Southwest Branch. The views expressed are those of the author and do not reflect the official policy or position of the Department of Defense or the U.S. Government. Approved for Public Release, Distribution Unlimited.

\section{References}

[1] J. Ramírez, J.C Segura, C. Benítez, A. de la Torre, and A. Rubio, "Efficient voice activity detection algorithms using long-term speech information", Speech Communication., 42, 271-287, 2004.

[2] J. Ramírez, P. Yelamos, J.M. Gorriz, and J.C. Segura (2006) "SVM-based speech endpoint detection using contextual speech features", Elec. Lett., 42(7), 2006. 
[3] S.G. Tanyer and H. Özer, "Voice Activity Detection in Nonstationary Noise", IEEE Trans. Speech Audio Process., 8(4), 478-482, 2000.

[4] A. Davis, S. Nordholm, and R. Togneri, "Statistical voice activity detection using low-variance spectrum estimation and an adaptive threshold", IEEE Transactions on Audio, Speech and Language Processing, Vol. 14, No. 2, 412-424, 2006.

[5] B.T. Meyer, S.V. Ravuri, M.R. Schadler, and N. Morgan, "Comparing Different Flavors of Spectro-temporal Features for ASR," Proc. Interspeech, 1269-1272, 2011.

[6] S.O. Sadjadi and J.H.L. Hansen, "Unsupervised speech activity detection using voicing measures and perceptual spectral flux," IEEE Signal Process. Letters, Vol.20, 197200, Mar. 2013

[7] M.J. Ross, H.L. Shaffer, A. Cohen, R. Freudberg, and H.J. Manley, "Average magnitude difference function pitch extractor," IEEE Trans. Acoust. Speech Signal Process., ASSP-22, 353-362, 1974.

[8] E. Scheirer and M. Slaney, "Construction and Evaluation of a Robust Multifeature Speech/Music Discriminator," Proc. IEEE ICASSP, Munich, Germany, , 1331-1334. 1997.

[9] L. N. Tan, and A. Alwan, "Multi-Band Summary Correlogram-Based Pitch Detection for Noisy Speech", accepted to Speech Communication.

[10] B.-S. Lee and D. Ellis, "Noise Robust Pitch Tracking by Subband Autocorrelation Classification," Proc. Interspeech-12, Portland, September 2012, paper P3b.05.

[11] T. Ng, B. Zhang, L. Nguyen, S. Matsoukas, X. Zhou, N. Mesgarani, K. Vesely and P. Matejka, "Developing a Speech Activity Detection System for the DARPA RATS Program", Proc. of ISCA Interspeech, 2012.

[12] K. Walker and S. Strassel, "The RATS Radio Traffic Collection System," Proc. of ISCA Odyssey, 2012. 